\title{
L'INFORMATIZZAZIONE DELL'ATLANTE LINGUISTICO SONORO ALD I (ATLANTE LINGUISTICO DEL LADINO CENTRALE E DIALETTI LIMITROFI I)
}

\section{CENNI INTRODUTTIVI}

L'ALD sonoro ${ }^{1}$ rappresenta, all'interno del progetto di ricerca ALD I, il settore più innovativo in merito alle strutture informatiche. Si tratta infatti dell'incorporazione di tutti i dati acustici (raccolti sul campo) in una delle varie catene di pubblicazione. Ciò comporta non solo notevoli vantaggi materiali (conservazione della qualità dei dati sonori a lungo termine, accesso rapido alla realtà acustica e facile duplificazione dei risp. dati), ma anche un considerevole incremento delle attuali possibilità di trattamento strettamente linguistico dei dati, oltre al valore generale dell'intera base documentaria messa a disposizione della ricerca diacronica dei secoli futuri.

La rete d'esplorazione di ALD copre gran parte delle varie regioni dell'Italia settentrionale che stanno attorno alla Ladinia dolomitica (Trentino/Alto Adige, Friuli, Veneto, Lombardia), nonchè, su territorio svizzero, il lato orientale dei Grigioni (Engadina, Valle Monastero). Per ulteriori dettagli si rinvia alla fig. 7. Il ns. interesse principale riguarda, come si vede dallo scioglimento della sigla ALD in italiano, il ladino centrale ${ }^{2}$, anche se $\mathrm{i}$ basiletti limitrofi vengono documentati in maniera analoga. In alcune isole linguistiche vengono inoltre rilevati i mesoletti romanzi $\left(\mathrm{L}_{2}\right)$ usati dagli autoctoni locutori germanofoni (cf. anche fig. 2).

Il questionario della prima parte del progetto ALD (= ALD I) comprende 806 domande d'ordine fonetico e morfosintattico (cf. Goebl/Kattenbusch/Stehl 1985). Le 216 inchieste di ALD I sono durate circa sei anni e mezzo (ottobre 1985 - aprile 1992). In tutto sono stati coinvolti cinque esploratori di origine, italiana, austriaca e tedesca, rispettivamente mista. L'archivio di ALD I (Istituto di Filologia Romanza, Università di Salisburgo) consta della documentazione scritta (216 questionari con le trascrizioni delle risposte basilettali effettuate in loco), della documentazione acustica (registrazione delle risposte su dispositivi magnetici), della documentazione fotografica (all'incirca 12.000 diapositive etno-fotografiche) e delle rispettive documentazio-

$1 \quad$ Il termine sonoro è stato scelto per evitare l'assonanza provocata dalla ns. originale denominazione italiana Atlante parlante. In tedesco ("Sprechender Sprachatlas") e in francese ("Atlas parlant") abbiamo però mantenuto l'elemento parlante.

2 Terminologia di G.I. ASCOLI (1873) corrispondente al termine tedesco Zentralrätoromanisch introdotto da Th. GARTNER (1883). 
ni elettroniche, di cui tratteremo qui sotto. Ogni anno di lavoro è stato accompagnato da una dettagliata relazione di lavoro retrospettiva, pubblicata sulla rivista scientifica sud-tirolese Ladinia, edita dall'Istitut Ladin "Micurà de Rü" di San Martin de Tor in Val Badia (cf. a questo proposito Kattenbusch/Goebl 1986, Szekely et al. 1987, Bauer et al. 1988, id. 1989, id. 1990, id. 1991, in corso di stampa ${ }^{3}$ ). É progettato continuare questa prassi sino al termine del progetto ALD I (1994/5).

In merito all'impostazione scientifica, l'ALD si definisce come atlante linguistico inter-regionale, seguendo il modello microscopico degli atlanti linguistici regionali francesi. Per il ns. sistema di trascrizione fonetica ci basiamo in gran parte sulle norme dell'atlante macroscopico AIS (cf. Jaberg/Jud 1928-1940; id. 1928, 24-36). La bipartizione dell'intero progetto in ALD I (fonetica e morfosintassi elementare) e ALD II (lessico e morfosintassi elaborata) risale ai modelli dei dizionari nazionali svizzeri e cioè GPSR, DRG e VSI. A questa generale progettazione logistica di ALD va aggiunta, come si è già accennato, l'integrazione innovativa della documentazione sonora ed etno-fotografica, che verranno - entrambi - trattate in catene additive di pubblicazione (cf. anche Bauer/Goebl 1991, 76).

Il finanziamento del progetto viene realizzato esclusivamente con fondi terzi (ted. Drittmittel), ricorrendo alle periodiche sovvenzioni da parte del CNR austriaco (FWF, Vienna), del Ministero alla Pubblica Istruzione (Vienna), del governo nord-tirolese (Innsbruck), degli Istituti Culturali Ladini "Micurà de Rü” (San Martin de Tor, Val Badia (BZ)) e "Majon de Fashegn" (Vich/Vigo di Fassa, (TN)) e della fondazione PRO HELVETIA (Svizzera).

\section{LA PROGETTAZIONE INFORMATICA GENERALE DI ALD I}

Può parere quasi superfluo sottolineare nuovamente l'importanza incontestabile dell'informatica per lo sviluppo della ricerca linguistica generaliter e per quella geolinguistica e dialettologica specialiter. L'istituzione della nuova disciplina linguistica computazionale $\left(\mathrm{CL}^{4}\right)$ che ha dato vita a vari sub-settori, tra cui la geolinguistica computazionale, può essere uno degli indici più significativi (si veda a questo proposito l'esemplare manuale di Bátori/Lenders/Putschke (1989) che presenta su ben 800 pagine 65 articoli su teoria, metodi e campi di applicazione di CL, nonchè una vasta bibliografia di altre 200 pagine; per la geolinguistica computazionale italiana cf. anche il contributo di Pennisi (1990) sul piano di informatizzazione dell'ALS).

Nell'ambito della ricerca dialettologica italiana si è, da circa $10 \mathrm{anni}$, iniziato qualche progetto geolinguistico semi- o totalmente informatizzato. In seno all'ALD si

3 Si vedano inoltre le regolari recensioni di Ladinia da parte del Festeggiato, pubblicate in questa sede (per i volumi IX (1985) - XII (1988) cf. ad es. Tekavčić 1987 e 1990).

4 La sigla CL deriva dalla denominazione anglo-americana Computational Linguistics (in ted. Computerlinguistik, in franc. linguistiquecomputationnelle, ma anche linguismatique, da linguistique + informatique). 
è, per la prima volta, realizzato un sistema automatico per il trattamento sia dei dati scritti sia di quelli sonori. La lingua parlata, indubbiamente l'oggetto più autentico e "prezioso" della ricerca geolinguistica in genere, costituisce quindi, assieme alla sua rappresentazione ausiliare tramite trascrizione fonetica (lingua scritta), la base di partenza non solo per la costituzione dell'atlante in folio e per quello sonoro, ma anche per ogni futuro studio dei materiali pubblicati.

\subsection{BREVE DESCRIZIONE DEL TRATTAMENTO ELETTRONICO DEI DATI SCRITTI}

Son le circostanze logistiche (di tempo, personale, fattibilità generale) che danno, nonostante le ns. posizioni "ideologiche" delineate prima, anzitutto preferenza alla realizzazione di un atlante linguistico tradizionale, cioè stampato su carta. Diamo alcune cifre esemplari che possono trasmettere la dimensione dell'immensa mole di lavoro per il solo primo volume di ALD (I):

A) INDICAZIONI TEMPORALI (in parte incroci tra i vari settori elencati):

a) predisposizione del progetto, rilevamenti di prova ecc.: 5 anni.

b) esplorazione di 216 PP.: 6 anni e mezzo.

c) sistema informatico (programmazione, immissione, correzione, emissione dati via PC): 7 anni.

d) stampa delle cartine: 2 anni.

B) INDICAZIONI QUANTITATIVE:

a) 216 punti d'esplorazione

$=216$ questionari

$=216$ banche dati di ca. 1.500 records ciascuna

$=$ ca. 324.000 singole risposte basilettali

=ca. 54 Megabytes di memoria

b) ca. 1.600 cassette magnetiche (C 90)

$=\mathrm{ca} .2 .400$ ore di registrazione

=ca. 648.000 singole risposte basilettali (due informatori per luogo d'inchiesta)

= ca. 16 Gigabytes di memoria (un informatore per luogo d'inchiesta)

c) ca. 12.000 diapositive etno-fotografiche

La fase iniziale del trattamento elettronico di ALD riguarda esclusivamente i dati del tipo B)a) riportati. Il primo ostacolo incontrato dall'informatico-geolinguista si presenta con il numero e con la varietà dei segni di trascrizione in uso. Il codice standardizzato dei Personal Computers (il cosiddetto ASCII ${ }^{6}$ ) prevede l'uso al massimo di 256 segni distinti nella sua versione elaborata. Pensando alle possibilità di

5 In senso di strategia politico-scientifica.

6 “American Standard Code for Information Interchange", valido per i cosiddetti PC compatibili-IBM (sistema operativo DOS). 
combinazione tra segni diacritici e segni di base di tutti i sistemi di trascrizione, si può facilmente intravedere la divergenza quantitativa tra l'offerta ASCII e la richiesta atlantistica. Il mercato mondiale di software mette a disposizione dell'utente certi prodotti, che permettono la rappresentazione di segni non inseriti nell'ASCI, tramite la rappresentazione grafica, adoperata spesso nei recenti sistemi di trattamento testi e di editoria elettronica. Questa prospettiva non si poteva però sfruttare per l'ALD, dato che avevamo, sin dall'inizio, scelto come sistema banca dati un prodotto (dBase III+) che non accetta la rappresentazione dei segni in modo grafico. La nostra scelta era sempre strettamente legata alla rispettiva diffusione del software a livello universitario internazionale, per permettere ad un numero più grande possibile di utenti l'accesso standardizzato ai dati elettronici di ALD.

La soluzione del problema di gestione elettronica dei dati di trascrizione è stata raggiunta in due fasi consecutive, tramite l'adoperamento di programmi, che permettono la codificazione di innumerevoli segni speciali attraverso combinazioni sostitutive di segni ASCII. Il primo prodotto (Lettrix) adattato alle esigenze di ALD I rendeva possibile, oltre alla gestione di tutti $\mathrm{i}$ dati in $\mathrm{dBase}$, l'emissione della trascrizione su stampanti ad aghi (cf. Bauer et al. 1988, 31-42; Bauer 1990a). Data l'apertura e la trasparenza del ns. sistema di codificazione, si potevano in seguito facilmente sostituire sia il programma di controllo (Lettrix $\rightarrow A L D-T e X^{7}$ ) sia il tipo di stampante (24 aghi $\rightarrow$ laser) (per ulteriori dettagli cf. Bauer et al. 1991, in corso di stampa). Le figg. 1 e 2 qui sotto, stampate ambedue attraverso $A L D$-TeX su una HP Laserjet III $^{8}$, rappresentano l'inventario attuale (1992) dei segni di trascrizione di ALD I (fig. 1) ed un esempio di emissione dalla banca dati delle trascrizioni ALD I (fig. 2).

\subsection{L'ATLANTE SONORO}

La necessità di mettere a disposizione dell'utente geolinguista una completa documentazione sonora di facile accesso (ed additiva alla documentazione scritta tradizionale) viene messa in rilievo, anche se indirettamente, da un articolo pubblicato recentemente sulla $\mathrm{ZrPh}$ (Wolf 1991). L'autore critica in maniera molto decisa la scarsa qualità delle trascrizioni fonetiche riscontrate in due pubblicazioni di geolinguistica sarda. Non è certo compito nostro riprendere in questa sede l'argomentazione di Wolf. Ci sembra invece molto utile mettere in evidenza, che un lavoro geolinguistico profondo ed obiettivo si può effettuare solo, se anche il ricercatore secondario ha la possibilità di ricorrere alla documentazione acustica originale, che sta alla base del lavoro descrittivo in questione ${ }^{9}$. Il caso sopraindicato ci sembra inoltre sintomatico

$7 \quad$ Il sistema $A L D-T e X$ si è potuto realizzare grazie ad una stretta ed amichevole collaborazione tra ALD e SSA (SüdwestdeutscherSprachatlas, "Atlante linguistico della Germania sud-orienta le", Università di Friburgo in Brisgovia; cf. anche Kelle/Schiltz in corso di stampa a e b).

8 Come introduzione generale al sistema TeX risp. LaTex si vedano Knuth (1986), Kopka (1991) e Lamport (1985).

9 Nel caso citato si tratta del disco (analogico) pubblicato assieme al volume 20 del Profilo dei dialetti 


\begin{tabular}{|c|c|c|c|c|c|c|c|c|c|c|c|}
\hline 1 & Courier & a & A & e & E & $\mathrm{i}$ & I & o & 0 & u & U \\
\hline 7 & ALD A & $a$ & $q$ & & & & & & $e$ & & \\
\hline 3 & ALD E & $e$ & & $e$ & $\xi$ & $\underline{e}$ & 8 & $\underline{e}$ & $\dot{\varepsilon}$ & & $\dot{\tilde{g}}$ \\
\hline 4 & ALD I & & & & & ? & $i$ & & & & \\
\hline 2 & ALD $O$ & $\stackrel{o}{n}$ & $\dot{o}$ & $\dot{\phi}$ & $\dot{o}$ & & $\tilde{\bar{\theta}}$ & 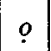 & $\varphi$ & & \\
\hline 5 & ALD U & $\bar{b}$ & & & & & $\check{y}$ & & $\bar{u}$ & $u$ & $u$ \\
\hline 6 & aldneu & $\alpha$ & $\alpha^{\prime}$ & 2 & á & $\grave{\partial}$ & $\tilde{s}$ & $\dot{\alpha}$ & $\overline{\dot{u}}$ & $\dot{u}$ & $\dot{u}$ \\
\hline 8 & ALTGR & $\grave{a}$ & & $i^{\prime}$ & & & & $\tilde{n}$ & & $\xi$ & \\
\hline
\end{tabular}

\begin{tabular}{|c|c|c|c|c|c|c|c|c|c|c|c|c|c|c|c|c|c|c|c|c|c|c|}
\hline 1 & Courier & b & c & d & $f$ & $\mathrm{~g}$ & h & $\mathrm{j}$ & k & 1 & $\mathrm{~m}$ & $\mathrm{n}$ & $\mathrm{p}$ & $q$ & $\mathrm{r}$ & $s$ & $\mathrm{t}$ & $\mathrm{v}$ & $w$ & $\mathrm{x}$ & $y$ & $\mathrm{z}$ \\
\hline 7 & ALD A & $\tilde{a}$ & $\bar{a}$ & 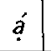 & $\grave{a}$ & áa & $\dot{a}$ & $\dot{\tilde{a}}$ & $\dot{\tilde{a}}$ & $\tilde{a}$ & $\bar{a}$ & $\dot{a}$ & $\overline{\bar{a}}$ & $\hat{a}$ & $\bar{a}$ & $\dot{a}$ & & & & & & \\
\hline 3 & ALD E & $\tilde{e}$ & $\bar{e}$ & $e ́$ & $\dot{e}$ & $\dot{\bar{e}}$ & $\dot{\bar{e}}$ & $\dot{\tilde{e}}$ & $\dot{e}$ & $\tilde{e}$ & $\bar{e}$ & $\dot{\bar{e}}$ & $\overline{\bar{e}}$ & $\dot{e}$ & $\overline{\bar{e}}$ & $\dot{e}$ & $\bar{e}$ & $\overline{\bar{e}}$ & $\dot{e}$ & $\dot{\bar{e}}$ & $\stackrel{\bar{e}}{e}$ & $\overline{\bar{e}}$ \\
\hline 4 & ALD I & $\tilde{\imath}$ & $\bar{\imath}$ & $i$ & $i$ & $\frac{1}{?}$ & $\stackrel{\grave{t}}{?}$ & $\hat{i}$ & $\dot{i}$ & $\tilde{\imath}$ & $\bar{\imath}$ & $\frac{1}{i}$ & & $\dot{\hat{\imath}}$ & & & & & & & & \\
\hline 2 & ALD O & $\tilde{\varphi}$ & $\bar{o}$ & $o$ & $\dot{\phi}$ & ó & $\dot{\bar{o}}$ & $\hat{o}$ & $\dot{\hat{o}}$ & $\tilde{o}$ & $\bar{o}$ & ó & $\tilde{o}$ & $\dot{o}$ & $\bar{o}$ & $\dot{o}$ & $\ddot{o}$ & $\bar{o}$ & $\dot{o}$ & $\dot{\bar{o}}$ & ó & $\overline{\bar{o}}$ \\
\hline 5 & ALD U & $\tilde{u}$ & $\bar{u}$ & $\dot{u}$ & $\grave{u}$ & $\overline{\bar{u}}$ & $\dot{\bar{u}}$ & $\tilde{\tilde{u}}$ & $\dot{\tilde{u}}$ & $\tilde{u}$ & $\ddot{u}$ & $\dot{\bar{u}}$ & $\overline{\bar{u}}$ & $\dot{\tilde{u}}$ & $\overline{\bar{u}}$ & $\dot{u}$ & $\ddot{u}$ & $\overline{\bar{u}}$ & $\dot{\tilde{u}}$ & $\dot{\vec{u}}$ & $\overline{\bar{u}}$ & $\overline{\bar{u}}$ \\
\hline 6 & aldneu & $\beta$ & $\dot{c}$ & $\delta$ & $\varphi$ & $g$ & $x$ & $\stackrel{\breve{s}}{s}$ & $\breve{c}$ & $l$ & $\ddot{z}$ & $\tilde{n}$ & $\hat{s}$ & $\check{g}$ & $r$ & $\stackrel{s}{s}$ & $\vartheta$ & $\hat{z}$ & $\underset{n}{u}$ & $x$ & $\stackrel{i}{i}$ & $z$ \\
\hline 8 & ALTGR & $\ddot{u}$ & $i$ & $i$ & $\grave{o}$ & $\dot{u}$ & & & & & & & & $a ́$ & $o ́$ & $\dot{e}$ & $\dot{u}$ & $\tilde{o}$ & $e ́$ & $\ddot{e}$ & & $\ddot{a}$ \\
\hline
\end{tabular}

\begin{tabular}{|c|c|c|c|c|c|c|c|c|c|c|c|c|c|c|c|c|c|c|c|c|c|c|}
\hline 1 & Courier & B & C & $D$ & $\mathrm{~F}$ & $\mathrm{G}$ & $\mathrm{H}$ & $\mathrm{J}$ & $\mathrm{K}$ & L & $M$ & $\mathrm{~N}$ & $P$ & $\mathrm{Q}$ & $\mathbf{R}$ & $\mathrm{S}$ & $\mathbf{T}$ & V & $\mathrm{W}$ & $\mathrm{X}$ & $\mathrm{Y}$ & Z \\
\hline 7 & ALD A & $\hat{a}$ & $\vec{a}$ & á & $\grave{a}$ & $\dot{a}$ & $\grave{a}$ & ấ & à̀ & $\tilde{\bar{a}}$ & $\tilde{a}$ & $\tilde{\tilde{a}}$ & & & & & & & & & & $\tilde{\hat{\theta}}$ \\
\hline 3 & ALD E & $\tilde{\xi}$ & $\bar{\varphi}$ & $\dot{\varepsilon}$ & $\dot{\varepsilon}$ & $\dot{\bar{e}}$ & $\dot{\bar{e}}$ & $\dot{\tilde{e}}$ & $\dot{\tilde{e}}$ & $\dot{\bar{\theta}}$ & $\tilde{\bar{e}}$ & $\tilde{\bar{e}}$ & $\tilde{\bar{e}}$ & & & & $\ddot{\varepsilon}$ & $\overline{\bar{\theta}}$ & $\dot{q}$ & $\dot{\bar{\varepsilon}}$ & $\overline{\bar{e}}$ & $\dot{\bar{e}}$ \\
\hline 4 & ALD I & $\tilde{\beta}$ & $\bar{q}$ & $i$ & $i$ & $\dot{\bar{l}}$ & $\frac{1}{i}$ & $\dot{\tilde{q}}$ & $\dot{\tilde{r}}$ & $\tilde{\bar{\eta}}$ & & $\hat{\bar{z}}$ & & & & & & & & & & \\
\hline 2 & ALD O & $\tilde{q}$ & $\bar{q}$ & $\phi$ & $\dot{\varphi}$ & $\bar{q}$ & $\dot{\bar{q}}$ & $\dot{\tilde{q}}$ & $\dot{\tilde{\varphi}}$ & $\dot{\tilde{\theta}}$ & & & $\ddot{0}$ & $\dot{o}$ & $\overline{\tilde{o}}$ & $\overline{\bar{\sigma}}$ & $\ddot{q}$ & $\overline{\bar{q}}$ & $\ddot{q}$ & $\dot{\hat{q}}$ & $\dot{\bar{\theta}}$ & $\overline{\hat{q}}$ \\
\hline 5 & ALD U & $\tilde{u}$ & $\bar{\mu}$ & $\hat{\psi}$ & $\dot{\psi}$ & $\overline{\bar{u}}$ & $\dot{\bar{u}}$ & $\dot{\tilde{u}}$ & $\dot{\tilde{u}}$ & $\dot{\bar{\psi}}$ & & & & & & & $\tilde{u}$ & $\overline{\bar{u}}$ & $\dot{\tilde{u}}$ & $\dot{\vec{u}}$ & $\overline{\overline{\tilde{u}}}$ & $\overline{\bar{u}}$ \\
\hline 6 & aldneu & $\dot{r}$ & $\ddot{c}$ & $d$ & $d$ & $\ddot{g}$ & $\bar{l}$ & $l$ & $\bar{n}$ & $l$ & $\bar{m}$ & $\eta$ & $\hat{s}$ & $\check{r}$ & $\hat{r}$ & $\breve{s}$ & $m$ & $\hat{z}$ & $\tilde{w}$ & $\gamma$ & $a ̊$ & $\check{z}$ \\
\hline
\end{tabular}

Fig. 1: Inventario dei segni di trascrizione di ALD I. Emissione attraverso programma ALD-TeX (aggiornato al mese di maggio 1992).

italiani sulla Sardegna (Pisa 1982). 


\begin{tabular}{|c|c|c|c|}
\hline \multirow{2}{*}{ domanda } & \multirow{2}{*}{ stimulus } & \multicolumn{2}{|c|}{ risposte } \\
\hline & & informatore 1 & informatore 2 \\
\hline 15.1 & l'amico & $d \alpha r m \bar{\imath} g \partial$ & - \\
\hline 16.1 & l'anatra & di ạ́nitra & - \\
\hline 28.1 & l'aquila & da ạ́kuila & $d a \stackrel{a}{a} g l$ \\
\hline 28.2 & le aquile & da ạkuilas & $d \partial \dot{a} g l s$ \\
\hline 33.1 & egli arriva & ear rovért & - \\
\hline 36.1 & l'asino & $d \alpha r k \dot{\bar{o} g \alpha} r$ & - \\
\hline 45.1 & il bagno & - & bạ́nio \\
\hline 49.1 & il barile & ạ lógol $\alpha$ & da bọta \\
\hline 61.1 & una borsa & - & ạ́na bọ́rsa \\
\hline 63.1 & la botte & dar kạrạtę́l & da bọ́ta \\
\hline 63.2 & le botti & da kạrạtẹlls & - \\
\hline 80.1 & cambiare & mudęrrn & - \\
\hline 109.1 & la cena & da tšặina & da tšậina \\
\hline 114.1 & il cerchio & $d \alpha r$ tšérki & - \\
\hline 114.2 & i cerchi & da tšéérklis & - \\
\hline 116.1 & il cervello & $d \alpha r$ tšęrvięi $i$ & dar tšęrvięi \\
\hline 116.2 & i cervelli & da tšęrviẹ́l & da tšęrviẹll \\
\hline 117.1 & il cervo & $d \alpha r$ tšérvo & - \\
\hline 123.1 & la chioccia & da klọtša & da klótšsia \\
\hline 123.2 & le chiocce & da klọtš̆inas & da klọtš̆ias \\
\hline 133.1 & la cipolla & da tšivọ́la & da tšivọ́la \\
\hline 133.2 & le cipolle & da tšivoólas & da tšivoólas \\
\hline
\end{tabular}

Fig. 2: Esempio di emissione banca dati ALD I attraverso programma ALD-TeX. Luogo d'inchiesta: Sauris/Zahre (UD) (isola linguistica germanofona). Risposte $=$ prestiti italiani risp. friulani in uso nel basiletto locale tedesco. (cf. a questo proposito anche Bauer et al. 1991, in corso di stampa). 
relativamente al ruolo ed all'importanza assegnati tradizionalmente alla trascrizione fonetica in sede di geolinguistica. La trascrizione è un sistema grafico ausiliare per la rappresentazione di realtà fonoacustiche, né più né meno.

L'alto standard dei mezzi tecnici odierni ci permette di rispondere in maniera più fruttifera alle esigenze di questa ns. scienza empirica. La lingua parlata si può registrare (non solo per scopi di archivio ${ }^{10}$ ), si può trattare elettronicamente, ordinare, catalogare, analizzare e rendere in fin dei conti facilmente accessibile a tutti (specialisti e profani). Quest'argomento a favore di lavori geolinguistici sonori vale tanto per il presente (e per un prossimo futuro) quanto per un futuro lontano. Le prospettive di uno studio diacronico da effettuarsi nel 3000 (o perchè no, nel 4000) vengono determinate dalla qualità e dalla completezza delle documentazioni che lasciamo in eredità alle future generazioni di dialettologi.

Con l'ALD I sonoro si è cercato di presentare per la prima volta un nuovo modello di atlanti linguistici. Il Personal Computer $\left(\mathrm{PC}^{11}\right)$ serve come strumento standard di gestione, sia dei dati scritti sia del corpus di dati acustici, i quali vengono riuniti ai vari tipi d'informazione meta-linguistica nella centrale banca dati ALD I (cf. qui sopra 2.1). II software dell'atlante sonoro consta di due moduli, uno di sviluppo (destinato cioè alla generazione di tutte le strutture basilari necessarie) ed uno di applicazione (in uso presso l'utente del prodotto finito).

\subsubsection{Il modulo di sviluppo}

La scelta di software per tutta l'impresa atlantistica sonora è stata dominata dal livello di diffusione internazionale dei prodotti in questione. Per la presentazione dell'ALD I parlante si è ricorso a Microsoft Windows (dapprima in versione 286, dal 1991 in poi in v. 3.0), che permette una divisione dello schermo in varie finestre nonchè il rispettivo accesso da parte dell' operatore via mouse (cioè senza bisogno di immettere comandi verbali attraverso la tastiera).

La programmazione del modulo di sviluppo (MS) è stata effettuata dalla ditta tedesca RST ${ }^{12}$ (cf. anche Bauer et al. 1990, 275-280), che è anche responsabile dell'intera configurazione del PC. Il MS serve alla digitalizzazione, al taglio elettronico e alla memorizzazione delle singole risposte basilettali, incise durante l'inchiesta su nastro magnetico. L'operatore collega un registratore (a nastri o cassette) al convertitore A/D-D/A (cf. nota 11) che, a sua volta, è attaccato ad una scheda speciale del PC. Dopo l'attivazione del MS Signal Editor ("editor di segnali") appare sotto il program-

$\overline{10}$ Il che in molti casi corrisponde a vasti cimiteri di dati!

11 Usiamo attualmente un PC dotato di: processore Intel 80386 (33 MHz), 4 Megabytes (MB) di RAM (= memoria centrale), $180 \mathrm{MB}$ di disco fisso (= memoria di massa), schermo Multisync (scheda grafica VGA, risoluzione $1024 \times 768$ punti per pollice), convertitore A/D-D/A (analogico/digitale-digitale/analogico). Per ulteriori dettagli si veda fig. 5.

12 c/o Univ.-Prof. Dr. Reinhard Köhler, Rechner- und Softwaretechnik, Kruppstraße 82, W-4300 Essen 1. 
ma di base (MS-Windows) un menu, dal quale si possono selezionare tre opzioni con le risp. sub-opzioni (cf. fig. 3).

\begin{tabular}{|lll|}
\hline Dater ("archivio") & Signal ("segnale") & INFo ("informazione") \\
\hline
\end{tabular}

1. ÖFFNEN ("aprire")

2. SPEICHERN ("memorizzare")

3. SPEICHERN UNTER ("memorizzare sotto")

4. ZUSAMMENFÜHREN ("unificare")

5. ENDE ("fine")
1. AUFNEHMEN ("registrare")
2. WIEDERGEBEN ("riprodurre")
3. Signaldaten ("dati segnale")
4. PEGEL ABGLEICHEN ("allineare scala")

1. INFO ÜBER SIGEDIT ("info editor segnali")

Fig. 3: Menu del modulo di sviluppo SigEdit ("editor segnali").

Il lavoro di preparazione del materiale acustico inizia con la sub-opzione registrare del menu segnale. Essa permette la digitalizzazione di un segnale sonoro contemporaneamente richiamato dal registratore analogico. Dopo sei secondi ${ }^{13}$ il programma interrompe il processo di digitalizzazione, facendo apparire sullo schermo l'oscillogramma della sequenza sonora elaborata (cf. fig. 4). Con l'opzione riprodurre è possibile riascoltare immediatamente il risultato del primo passo di digitalizzazione. L'opzione dati segnale serve all'immissione di varie informazioni relative al luogo d'inchiesta, al numero della domanda e così via. L'ultimo punto del menu segnale si riferisce all'intensità fonica del dato sonoro in questione. Attivando allineare scala il volume acustico viene alzato ad un livello standard, per garantire un ascolto lineare durante ogni futuro accesso (libero ed incrociato) alla banca dati acustica.

La fig. 4 presenta il risultato del lavoro appena descritto. Nella parte inferiore dello schermo si vede tutto l'oscillogramma con l'indicazione della risp. durata in millisecondi (ms). A sinistra ed a destra della curva si osservino due sbarre separatorie che possono essere spostate col mouse per delimitare una parte precisa dell'intero segnale. Così avviene il taglio elettronico della risposta basilettale grezza, avendo come risultato, dopo l'eventuale eliminazione di digressioni (voce dell'esploratore, rumori circostanti ecc.), il dato netto che va memorizzato nella banca dati. Nella parte superiore dello schermo viene rappresentata una sequenza parziale di $31 \mathrm{~ms}$ di lunghezza, offrente la possibilità di effettuare ulteriori tagli molto precisi.

13 Si tratta esattamente di 6.160 millisecondi. 


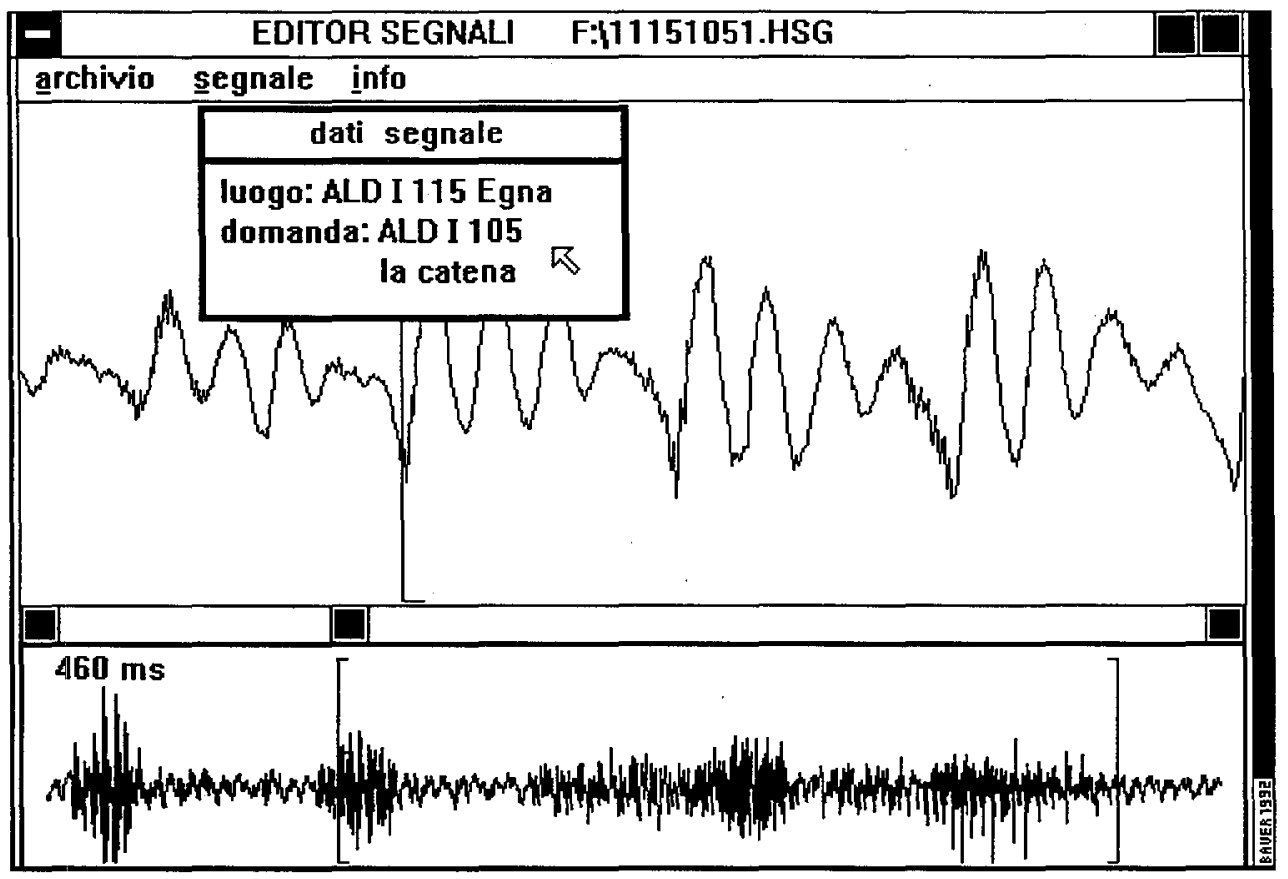

Fig. 4: Schermo modulo di sviluppo “editor segnali" (SigEdit versione 3.03).

L'opzione archivio mette a disposizione tutti i mezzi per la comunicazione tra programma e memoria di massa. Si possono aprire archivi già esistenti per effettuare eventuali modifiche o riascolti di controllo. Per memorizzare certi dati prestabiliti, si usa in genere la sub-opzione memorizzare sotto, che richiede l'immissione di un nome preciso per memorizzare l'archivio in questione. Nel caso dell'ALD I ad ogni file acustico viene assegnato un nome composto di otto numeri, che rappresentano la parte del progetto (1a posizione), il no. del luogo d'inchiesta (dalla 2a alla 4a pos.), il no. di domanda secondo il questionario ALD I (pos. 5-pos. 7) ed infine il no. della versione di risposta (pos. 8). Ogni archivio acustico porta inoltre l'estensione $H S G^{14}$. Per collegare $\mathrm{i}$ dati acustici ai dati scritti (i.e. trascrizioni), il nome di ogni file sonoro riappare in un campo della banca dati centrale.

La sub-opzione memorizzare dell'opzione principale archivio (sempre nel menu del modulo di sviluppo editor segnali, cf. fig. 3) serve a memorizzare una versione modificata di un archivio già esistente con lo stesso nome. Unificare significa combinare due o più archivi per crearne uno nuovo.

La terza opzione principale fornisce l'utente di una serie di informazioni relative alla versione del programma in uso nonchè al copyright ${ }^{15}$.

14 Cf. fig. 4, archivio 11151051.HSG: ALD I, luogo 115, domanda 105, versione 1. 


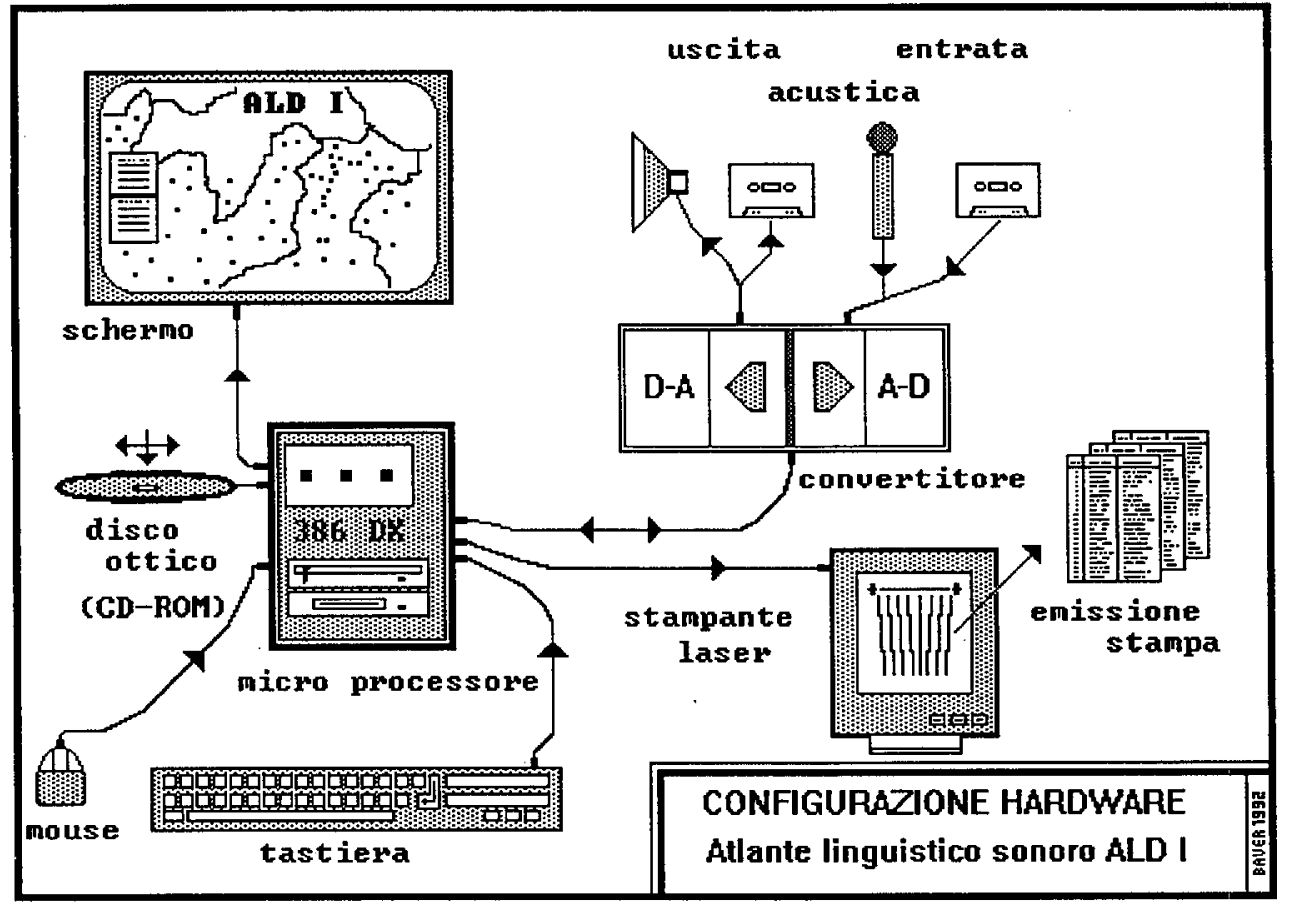

Fig. 5: Configurazione hardware dell'Atlante linguistico sonoro ALD I.

Secondo un ns. calcolo la preparazione di tutti i dati acustici ALD I (ca. 324.000 singole risposte) mediante il MS SigEdit richiede all'incirca 5.400 ore di lavoro ossia tra quattro e cinque anni, prendendo in considerazione le capacità realistiche di un operatore professionale.

\subsubsection{IL MODULO DI APPLICAZIONE}

Anche il modulo di applicazione (MA) dell'atlante sonoro è un programma compatibile con $M S$ Windows. La realizzazione del programma risale di nuovo alla sopraccitata collaborazione tra il progetto ALD e la ditta RST (cf. anche Bauer in corso di stampa, c). Il menu principale del MA (cf. fig. 6), le cui opzioni verranno descritte qui sotto, viene ugualmente caricato attraverso il mouse. Ciò garantisce un uso molto semplice e facilmente apprendibile anche da parte di linguisti non specializzati in informatica.

La prima opzione principale (atlante-caricare) mette a disposizione dell'utente tutti gli atlanti sonori inseriti nel sistema. L'ALD I sonoro attuale dispone di due versioni di prova, realizzate tra il 1990 ed il 1991.

15 Stato attuale (maggio 1992): SigEdit versione 3.03, (c) RST 1989-1991. 


\begin{tabular}{|llll|}
\hline Atuas ("atlante") $\quad$ AusWAHL ("scelta") & ABSPIELEN ("ascolto") & INFO \\
\hline
\end{tabular}

1. LADEN ("caricare")

2. ENDE ("fine")

1. ORTSLISTE ("elenco luoghi")
2. STIMULuSLISTE ("elenco stimuli")

1. NACH ORTEN ("secondo luoghi")

2. NACH STIMULI ("secondo stimuli")

3. OPTIONEN ("opzioni")

3.1. STIMULUSWIEDERHOLUNGEN ("ripetizioni stimuli")

3.2. Pausenlänge (MS) ("lunghezza pausa" (ms))

3.3. AusGaBE ("emissione")

1. INFO ÜBER SPRACHATLAS ("info atlante linguistico")

Fig. 6: Menu del modulo di applicazione dell'Atlante linguistico sonoro ALD I.

La versione di prova 1 (1990) comprende 13 luoghi d'inchiesta della rete di ALD I (Val di Sole, Val di Non, Ladinia Dolomitica) e 25 domande (da ALD I 100 la carne a ALD I 125 il cibo/i cibi). Prendendo in considerazione tutte le versioni paradigmatiche delle risposte (forme maschili e femminili, singolare e plurale) si tratta di una media di 60 singole risposte basilettali per ogni luogo d'inchiesta. La matrice dei dati della versione di prova no. 1 contiene dunque ca. 780 unità (13 luoghi x 60 risposte). Per una descrizione più dettagliata si veda Bauer in corso di stampa, a.

La seconda versione di prova (1991) è stata realizzata dapprima in edizione-PC col sistema informatico di cui trattiamo in questa sede. In seguito si è pubblicata un'edizione popolare sotto forma di un disco compatto audio-digitale (i.e. da ascoltare con un tradizionale riproduttore-CD). Il CD - ALD I (Bauer 1991b) raduna 78 luoghi d'inchiesta (dai Grigioni fino al Cadore) con tre stimuli scelti per il loro valore documentario in senso di fonetica storica (evoluzione dei nessi latini CA-e CL- in: ALD I 105 la catena, ALD I 119 egli chiama, ALD I 122 la chiesa). La fig. 7 ne rappresenta uno schermo esemplare, ripreso nel momento dell'ascolto della risposta basilettale per ALD I 105 la catena nel luogo d'inchiesta 65 - San Martin de Tor, Val Badía (BZ). La matrice dei dati della versione di prova no. 2 contiene ca. 390 unità (78 luoghi x, in media 5 risposte). Sul CD l'accesso ai dati si effettua tramite il richiamo di uno dei 98 indirizzi ivi incisi. Ogni singolo indirizzo rappresenta o un luogo d'inchiesta (con le risp. risposte) o un sentiero acustico già prestabilito secondo criteri geolinguistici ben precisi. All'interno di ogni indirizzo si può inoltre accedere a un numero di indici (fino a 99 indici/indirizzo). La matrice dei dati del CD, composta da indirizzi ed indici 
comprende 849 unità acustiche. Il CD viene accompagnato da un opuscolo bilingue (italiano e tedesco) e dalle tre cartine in questione! 16.

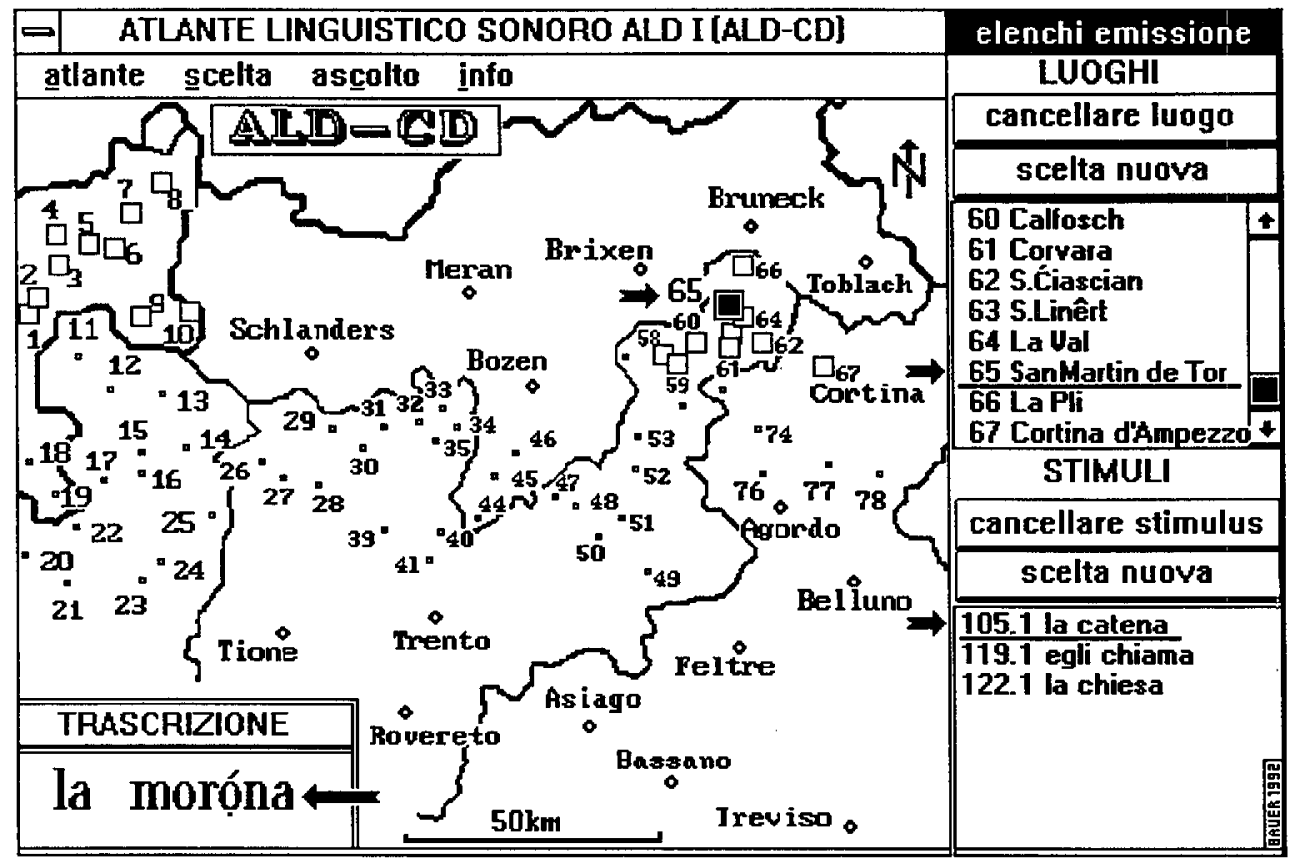

Fig. 7: Schermo modulo applicazione Atlante linguistico sonoro ALD I.

Esempio CD - ALD I:

luogo scelto: 65 San Martin de Tor; domanda scelta: ALD I 105 la catena.

Oltre a queste due versioni pilota si sono realizzati altri due moduli con dati non appartenenti all'ALD. Nel primo caso (1991) si tratta dell'elaborazione di un piccolo corpus acustico di italiano regionale (9 luoghi x 5 stimuli, cf. Bauer in corso di stampa, b), nel secondo caso (1992) si è messa in azione una versione di prova di un futuro EHHA sonoro (Euskal-Herriko Hizkuntz-Atlasa, "Atlas linguistique du Pays Basque") assieme agli autori baschi.

Tornando al menu principale del modulo di applicazione (cf. fig. 6) si osservino i due elenchi (per luoghi e stimuli) nell'opzione scelta, che permettono un libero accesso ai dati rispettivi nonchè una prima determinazione dell'ordine d'emissione. I luoghi d'inchiesta si possono anche caricare (i.e. scegliere e mettere negli elenchi

16 Per eventuali richieste indirizzarsi a: Progetto di ricerca ALD I, Institut für Romanistik, Akademiestraße 24, A-5020 Salisburgo. Prezzo di costo = prezzo di vendita per $C D-A L D I+$ booklet bilingue (44 pp.) + estratto (45 pp.) + 3 cartine ingrandite (formato A3): Lit. 10.000,-- / DM 15,-- / ös $100,--$. 
emissione, cf. fig. 7, finestre a destra dello schermo) direttamente sulla cartina, cliccando col mouse nei piccoli quadrati accanto ai risp. numeri dei luoghi d'inchiesta. L'effetto della scelta dei punti (sia dopo l'attivazione nell'elenco luoghi sia dopo l'accesso diretto sulla cartina attraverso il mouse) è sempre lo stesso: tutti i luoghi d'inchiesta vengono rappresentati come attivi sulla cartina stessa (tramite quadrati rossi accanto al numero del luogo ${ }^{17}$ ) e nell'elenco di emissione luoghi (indicazione del numero e del nome del luogo scelto). Gli stimuli scelti appaiono ugualmente nell'elenco di emissione stimuli (indicazione del numero della domanda e del lemma).

La terza opzione principale (ascolto) permette l'uso vero e proprio del modulo. L'ordine di emissione viene determinato dalle sub-opzioni secondo luoghi (il che corrisponde all'ascolto sequenziale di una serie di studi monografici dialettali locali) e secondo stimuli (il che corrisponde alla consultazione acustica di una cartina d'atlante linguistico). Durante l'ascolto il quadrato del luogo attivato viene rappresentato in verde $^{18}$ sulla cartina, i rispettivi nomi di luogo e domanda vengono evidenziati negli elenchi di emissione (sottolineati nella fig. 7). Nell'angolo inferiore a sinistra dello schermo appare una finestra che fa vedere la trascrizione fonetica della risposta in ascolto. Così l'utente riceve contemporaneamente tutte le informazioni scritte (relative al luogo d'inchiesta, alla domanda ed alla trascrizione) e quelle sonore rispettive.

L'attivazione delle opzioni del punto-menu ascolto mette a disposizione un altro gruppo di parametri per determinare il modo di ascolto (cf. fig. 6, punti 3.1.-3.3.). Ripetizioni stimuli serve a regolare il numero delle ripetizioni di ogni singola risposta durante l'ascolto. Lunghezza pausa permette l'inserimento di pause (da 0 fino a 10.000 millisecondi) tra un'emissione-risposta e l'altra. Emissione infine provoca una riduzione dei dati prescelti (i.e. messi negli elenchi di emissione) tramite il marcaggio di singoli dati.

L'opzione info è analoga a quella descritta per il modulo di sviluppo. Stato attuale: SprachAt, versione 3.02, (c) RST 1989-1991.

Per ragioni di spazio non possiamo trattare tutti i moduli dell'atlante sonoro in maniera dettagliata. Il programma globale mette ancora a disposizione altri arnesi elettronici, che servono ad es. alla rappresentazione dei segni fonetici sullo schermo (modulo FontEdit), alla determinazione delle coordinate dei punti d'inchiesta sulla cartina (modulo RST-Position) o alla progettazione grafica della cartina stessa (PaintShow Plus, MS-Paintbrush, ecc.). Va ancora una volta messo in rilievo, che tutti gli strumenti elettronici sviluppati per il progetto ALD I possono facilmente essere adattati ad ogni altro tipo di progetti e dati geolinguistici.

Le prospettive di pubblicazione di ALD I prevedono i segg. quattro tipi di documentazione:

17 Sulla fig. 7 (per ragioni di tipografia in bianco e nero) i quadrati rossi in questione (cf. ad es. PP. 45, $46,47 \mathrm{ss}$.) sono sostituiti da quadrati bianchi (vuoti).

18 Sulla fig. 7 (per ragioni di tipografia in bianco e nero) il quadrato verde in questione (P. 53, Vich/Vigo di Fassa) è sostituito da un quadrato nero. 
1. atlante cartografico stampato in maniera "tradizionale" con vari indici in aggiunta (previsto per 1994-1995),

2. banca dati elettronica relativa ai dati di 1) su dispositivo magnetico o ottico-digitale (previsto per 1994-1995),

3. atlante sonoro su dispositivo ottico-digitale (CD-ROM, WORM) (data di pubblicazione non ancora fissata),

4. documentazione etno-fotografica su dispositivo ottico-digitale (CD-ROM, WORM) (data di pubblicazione non ancora fissata).

\section{BIBLIOGRAFIA}

AIS: Jaberg, Karl / Jud, Jakob (eds.) (1928-1940): Sprach- und Sachatlas Italiens und der Südschweiz. Zofingen, 8 voll.

Ascoli, Graziadio Isaia (1873): Saggi ladini, in: Archivio glottologico italiano 1, LVI, 1-556. Ristampa: Torino 1972.

Bátori, István S. / Lenders, Winfried / Putschke, Wolfgang (eds.) (1989): Computational Linguistics - Computerlinguistik. An International Handbook on Computer Oriented Language Research and Applications - Ein internationales Handbuch zur computergestützten Sprachforschung und ihrer Anwendungen. Berlin-New York, (= HSK, 4).

Bauer, Roland (1990a): Convenzioni di codificazione-dati per l'ALD I / Kodieranweisungen für die Dateneingabe zum ALD I. Versione / Version 1.4. Salisburgo, con un'aggiunta in merito alla correzione dei dati / mit einer Ergänzung zur Datenkorrektur (1992), 25 pp., [dattil.].

Bauer, Roland (1990b): 4. Transkriptionsseminar zum ALD I, in: Europa Ethnica 47/2, 100.

Bauer, Roland (1991a): 5. Transkriptionsseminar zum ALD I, in: Europa Ethnica 48/1, 37-38.

Bauer, Roland (1991b): CD - ALD I/ALD I - CD, 98 campioni fonici per l'ALD I/98 Tonproben zum ALD I. Salisburgo, disco compatto con booklet bilingue (ital./ted.), 44 pp.

Bauer, Roland (in corso di stampa, a): Neue Perspektiven der LDV im Forschungsprojekt ALD I - Der sprechende Sprachatlas, in: Viereck, Wolfgang, op.cit.

Bauer, Roland (in corso di stampa, b): Ein Sprach-Atlas beim Wort genommen: $A L D$ I, der "Sprechende", in: WINKELMANN, Otto (ed.), Stand und Perspektiven der romanischen Sprachgeographie, Wilhelmsfeld, (= pro lingua).

Bauer, Roland (in corso di stampa, c): Die romanische Geolinguistik im Spannungsfeld zwischen Wirtschaft und Wissenschaft: Kooperationsmodelle im Projekt ALD I, in: RADTKE, Edgar/THUN, Harald (eds.), Neue Wege der romanischen Geolinguistik, Francoforte, (= Dialectologia Romanica Pluridimensionalis, 1). 
Bauer, Roland (in corso di stampa, d): Strumenti e metodi di rilevamento per la raccolta dei dati di ALD I, in: RADTKE, Edgar/THUN, Harald (eds.), op.cit.

Bauer, Roland / Goebl, Hans (1991): Presentazione di ALD I - Atlante linguistico del ladino dolomitico e dialetti limitrofi, parte prima, in: Per Padre Frumenzio Ghetta, O.F.M., Scritti di storia e cultura ladina, trentina, tirolese e nota bio-bibliografica in occasione del settantesimo compleanno, a cura della Biblioteca Comunale di Trento e dell'Istitut Cultural Ladin, Trento/Vich-Vigo di Fassa, 7399.

Bauer, Roland / Gislimberti, Silvio / Perini Elisabetta / Szekely, Tino / Goebl, Hans (1988): Arbeitsbericht 3 zum ALD I - Relazione di lavoro 3 per l'ALD I, in: Ladinia XII, 17-56.

Bauer, Roland / Goebl, Hans / Szekely, Tino / Gislimberti, Silvio / Perini, Elisabetta (1989): Arbeitsbericht 4 zum ALD I - Relazione di lavoro 4 per l'ALD I, in: Ladinia XIII, 185-229.

Bauer, Roland / Böhmer, Helga / Gislimberti, Silvio / Goebl, Hans / Köhler, Reinhard / Schleusser, Martin / Szekely, Tino / Tyroller, Hans (1990): Arbeitsbericht 5 zum ALD I - Relazione di lavoro 5 per l'ALD I, in: Ladinia XIV, 259-304.

Bauer, Roland / Böhmer, Helga / Goebl, Hans / Haimerl, Edgar / Schiltz, Guillaume / Tyroller, Hans (in corso di stampa): Arbeitsbericht 6 zum ALD I, in: Ladinia XV (1991).

DRG (1939-1946ss.): Dicziunari rumantsch grischun publichà da la Società Retorumantscha. Cuoira/Chur.

Gartner, Theodor (1883): Raetoromanische Grammatik. Heilbronn. Ristampa: Vaduz-Liechtenstein 1984.

Goebl, Hans (1978): Ein Sprach- und Sachatlas des Zentralrätoromanischen (ALD), in: Ladinia II, 19-33.

Goebl, Hans (1990): Vorstellung des "Sprachatlasses des Zentralrätoromanischen und annexer Gebiete / Atlante linguistico del ladino centrale e dialetti limitrofi" (ALD I), in: Klagenfurter Beiträge zur Sprachwissenschaft 15-16, 130-135.

Goebl, Hans (in corso di stampa): L'atlas parlant dans le cadre de l'Atlas linguistique du ladin central et des dialectes limitrophes, in: Atti del "Nazioarteko dialektologia biltzarra / Congreso internacional de dialectología", Bilbo/Bilbao 21.25.10.1991.

Goebl, Hans / Bauer, Roland (in corso di stampa): L'atlante linguistico del ladino centrale e dialetti limitrofi (ALD I): Stato attuale dei lavori, in: RUFFINO, Giovanni (ed.), Atlanti linguistici italiani e romanzi, Esperienze a confronto, Palermo 3.-7.10.1990.

Goebl, Hans / Kattenbusch, Dieter / Stehl, Thomas (1985): Fragebuch zum ALD I Questionario per l'ALD I. Salisburgo, 1987 (2. ediz.corr.), 1988 (3. ediz.). 
GPSR: Gauchat, Louis / Jeanjacquet, Jean / Tappolet, Ernest (1924-1933ss.): Glossaire des patois de la Suisse romande. Neuchâtel-Paris.

Jaberg, Karl / Jud, Jakob (1928): Der Sprachatlas als Forschungsinstrument. Kritische Grundlegung und Einführung in den Sprach- und Sachatlas Italiens und der Südschweiz. Halle.

Jaberg, Karl / Jud, Jakob (1987): L'atlante linguistico come strumento di ricerca. Fondamenti critici e introduzione. Edizione italiana a cura di G. Sanga. Milano 1987.

Kattenbusch, Dieter / Goebl, Hans (1986): Die ersten Enqueten für den ALD I Erfahrungen und Ergebnisse (ALD-Arbeitsbericht 1), in: Ladinia X, 5-32.

Kelle, Bernhard / Schiltz, Guillaume (in corso di stampa, a): Die Wiedergabe phonetischer Schriftzeichen in der automatisierten Sprachatlas- und Druckvorlagenherstellung (TeuTeX), in: VIERECK, Wolfgang (ed.), op.cit.

Kelle, Bernhard / Schiltz, Guillaume (in corso di stampa, b): Die Darstellung phonetischer Transkription, in: Atti della "10. Tagung der deutschsprachigen TeX-Interessenten", Vienna, 20.-22.2.1991.

Knuth, Donald E. (1986): The TeX book. Reading/MA.

Kopka, Helmut (1991): LaTex - Eine Einführung. Bonn-München.

Lamport, Leslie (1985): LaTeX - A Document Preparation System. Reading/MA.

Lesina, Roberto (1991): Software \& Hardware. Dizionario dei termini informatici italiano-inglese inglese-italiano. Bologna.

Pennisi, Antonino (1990): Piano di informatizzazione dell'Atlante linguistico della Sicilia (ALS). Fondamenti, strategie, progetto, in: RUFFINO, Giovanni (ed.), Materiali e ricerche dell'Atlante linguistico della Sicilia, Vol. I, Palermo, 3-106 (estratto).

Quartiroli, Ivo (1990): Dizionario Acronimi \& Termini di informatica. Milano.

Szekely, Tino / Perini, Elisabetta / Gislimberti, Silvio / Goebl, Hans (1987): Arbeitsbericht 2 zum ALD I - Relazione di lavoro 2 per l'ALD I, in: Ladinia XI, 183-218.

Tagliavini, Carlo (1965): La corretta pronuncia italiana. Corso discografico di fonetica e ortoepìa. Bologna.

Tekavčić, Pavao (1987): Recensione di Ladinia IX-X (1985-1986), in: Linguistica XXVII, 180-185.

Tekavčić, Pavao (1990): Recensione di Ladinia XI-XII (1987-1988), in: Linguistica XXX, 224-228.

Viereck, Wolfgang (ed.) (in corso di stampa): Atti di "Internationaler Dialektologenkongreß", Bamberg, 29.07.-4.8.1990, Wiesbaden, (= Beihefte der Zeitschrift für Dialektologie und Linguistik).

VSI (1952-1965 ss.): Vocabolario della Svizzera italiana. Lugano.

Wolf, Heinz Jürgen (1991): Orgosolo und das dialektologische Ungemach, in: Zeitschrift für romanische Philologie 107, 411-417. 